\title{
SEGREGATION MEASURES FOR DIFFUSION-CONTROLLED A+B REACTIONS
}

\author{
L.A. HARMON, L. LI, L.W. ANACKER and R. KOPELMAN \\ Chemistry Department, The University of Michigan, Ann Arbor, MI 48109, USA
}

Received 30 June 1989; in final form 12 September 1989

\begin{abstract}
New measures of segregation are defined and calculated for the $A+B \rightarrow 0$ reaction on one-dimensional and fractal lattices (Sierpinski carpet and gasket). We differentiate between local and global segregation parameters. Kesults and comparisons are given for steady-state simulations that strictly conserve the equality of A and B particle densities. This work demonstrates that global kinetic laws are related to both local and global segregation.
\end{abstract}

\section{Introduction}

Diffusion-controlled elementary binary A+B reactions on fractal and low-dimensional structures exhibit anomalous (non-classical) kinetics [1-6]. Furthermore, the reactant populations have been observed to segregate, forming regions or aggregates of a single type. For transient reactions, this was discovered theoretically by Ovchinnikov and Zeldovich [1] (dimensions 1 to 3), was simulated by Toussaint and Wilczek [3] (dimensions 1 and 2) and was demonstrated for fractal dimensions by Blumen et al. [4] (Sierpinski gasket). Since reactions between the same species do not occur $(A+A$ or $B+B)$, the existence of segregation restricts reactions to cluster boundaries. By this means, segregation can dramatically alter the effective topology of the reaction medium. Even more surprising has been the discovery of segregation in steady-state reactions [5-8], in which reactants are added at a constant rate until a steady-state reaction rate (and population) has been established. In both cases, the degree of segregation has been found to increase with decreasing spectral dimension, $d_{\mathrm{s}}$. Recent work has suggested that $d_{\mathrm{s}}$ is a critical factor in determining the degree of reactant segregation [9].

Systematic study of the relationships between the topology of the reaction medium, reactant segregation, and reaction kinetics, requires quantitative methods to characterize particle segregation. Distinguishing random fluctuations from genuine segre- gation is difficult, even in relatively simple systems. Criteria are needed for consistent, quantitative, comparison of particle distributions during reactions in systems with a variety of topologies and densities. Several such parameters are discussed here and used to describe the degree of segregation at steady state for reactions on a one-dimensional lattice $\left(d_{s}=1\right)$, the Sierpinski carpet $\left(d_{\mathrm{s}}=1.68\right)$ and the Sierpinski gasket $\left(d_{2}^{\prime}=1.36\right)$.

\section{Simulation methodology}

In steady-state simulations, the initial population of reactants on the lattice is zero. Equal numbers of $A$ and $B$ are added at regular time intervals, their positions chosen at random. If an attempt is made to add a particle to a site occupied by a particle of the other type, either the particles can react or a new (random) site can be selected. The former process is referred to as landing with "vertical reaction", while the latter is termed landing without "vertical reaction". Each particle is moved with probability $z^{-1}$ to one of its $z$ nearest-neighbor sites on each time step. Only one particle is allowed to occupy a lattice site. Reaction occurs when an A particle attempts to move to a site occupied by a $B$ particle or vice versa. The details of the simulations have bcen described elsewhere for the 1-D lattice [10], the Sierpinski carpet [11], and the Sierpinski gasket [5].

Landing with vertical reaction was used in simu- 
lations on the 1-D lattice and Sierpinski carpet. No vertical reaction was allowed on the Sierpinski carpet. It has been shown elsewhere [12] that the type of landing process used has a significant effect on the development of segregation in reacting systems. Thereforc, results from the two types of simulation cannot be compared on a quantitative basis.

\section{Segregation parameters}

Two categories of segregation parameters can be identified: local and global [13]. Local parameters are sensitive to the environments experienced by individual reactants. For this reason, they are particularly informative about the conditions which determine instantaneous reaction kinetics. Global parameters, on the other hand, describe overall particle distributions. As such, they are useful for characterizing and monitoring the evolution of steady states.

\subsection{Iocal segregation parameters}

A convenient basis for defining local segregation parameters is the relative numbers of nearest-neighbor (adjacent, dimer) pairs which are matched ( $A$ A or B-B) or mixed (A-B or B-A) [11,12]. One such parameter is given by

$P_{\mathrm{NN}}=\frac{N_{\mathrm{AA}}+N_{\mathrm{BB}}-\left(N_{\mathrm{AB}}+N_{\mathrm{BA}}\right)}{N_{\mathrm{AA}}+N_{\mathrm{BB}}+N_{\mathrm{AB}}+N_{\mathrm{BA}}}$

in which $N_{\mathrm{AA}}, N_{\mathrm{BB}}, N_{\mathrm{AB}}$ and $N_{\mathrm{BA}}$ are the numbers of A-A, B-B, A-B and B-A pairs, respectively. In a totally random system, the numbers of matched and mixed pairs are equal and $P_{\mathrm{NN}}$ is 0 . In a totally segregated system, the number of mixed pairs is approximately 0 , yielding $P_{\mathrm{NN}}=1$. A completely ordered, or super-lattice, structure with a super-lattice spacing of 1 (density $=1$ ), yields $P_{\mathrm{NN}}=0$ since the number of mixed and matched pairs is equal. For super-lattice spacings greater than the nearest neighbor separation, there are no nearest neighbor pairs and $P_{\mathrm{NN}}$ is undefined.

Fig. 1 shows $P_{\mathrm{NN}}$ as a function of time for the steady-state reaction on a one-dimensional lattice. The total population density is also presented as a function of time, scaled to fit on the same plot. Al-

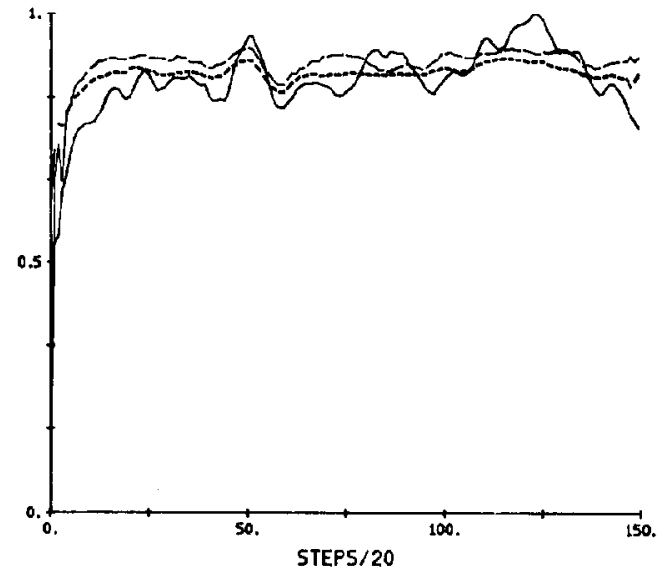

Fig. 1. Approach to the steady state: number of walkers and segregation parameters - 1-D. The total number of walkers, $N$ (solid curve), and segregation parameters $P_{\mathrm{b}}$ (long dashes) and $P_{\mathrm{CN}}$ (short dashes) are plotted as a function of time as described in section 3 of the text. Two walkers were added per step to a 128site lattice (with vertical annihilation and cyclic boundary conditions). Data were averaged over 10 time steps and smoothed. The total number of walkers was scaled to fit on the plot.

though there are significant fluctuations in $P_{\mathrm{NN}}$ at longer times, the segregation measured by this criterion is seen to reach a stable level at early times. The degree of segregation closely follows the time development of the density. The steady-state value of $P_{\mathrm{NN}}$ is approximately 0.9 , corresponding to nearly total segregation.

The expression of eq. (1) generalizes to higher-dimensional and fractal lattices as long as a taxicab [14] definition of "ncarest neighbor" is employed, restricting neighbors to sites connected by the underlying lattice (fig. 2a). The time development of $P_{\mathrm{NN}}$ is shown in fig. 5 for a steady-state simulation on a fifth-order Sierpinski carpet. The steady-state value of $P_{\mathrm{NN}}$ is approximately 0.7 , which indicates substantial segregation, although less than the value for the 1-D lattice. The value of $P_{\mathrm{NN}}$ after $10^{6}$ time steps on an eight-order Sierpinski gasket is included as a single point. In this system, with $P_{\mathrm{NN}}=0.87$ the segregation is nearly complete.

\subsection{Global segregation parameters}

In searching for appropriate parameters by which to describe the global spatial distribution of particles 

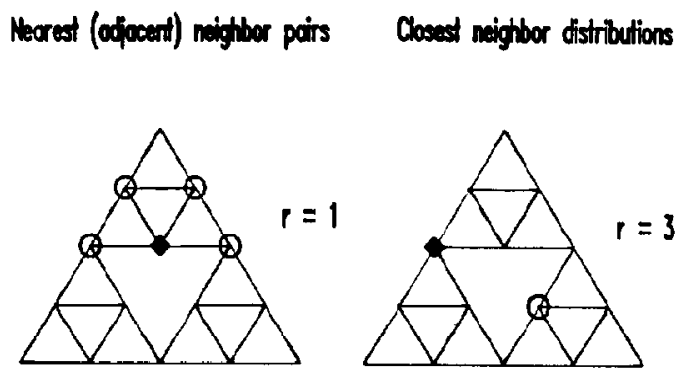

Fig. 2. Taxicab geometries. (a) The nearest neighbors to the solid diamond are marked with open circles. (b) The closest neighbor to the solid diamond is marked with an open circle (assuming no other lattice sites are occupied). The taxicab cistance, $r$, between these two sites is 3 .

on a lattice, it is necessary to move beyond nearest neighbor relationships. One way to view segregation is in terms of the formation of clusters or aggregates of a single particle type. Parameters which characterize cluster structures, e.g. the sizes and spatial extents of clusters, can then be used to characterize particle distributions as a whole.

In one-dimensional systems, clusters are easily defined: a cluster is a region in which the particles are all of one type ( $\Lambda$ or $B$ ), bounded on both ends by particles of the opposite type. One quantity which describes such clusters is the number of boundaries between clusters, $N_{\mathrm{b}}$, which is equal to the number of clusters. Since reactions can only occur at the boundaries between clusters, $N_{\mathrm{b}}$ describes the number of reactive regions. On the average, the ratio of the total number of particles $(N)$ to $N_{\mathrm{b}}$, gives the number of particles in each cluster. The average cluster length (extent) is found by dividing the number of clusters by the total length of the lattice.

A global segregation parameter can be formed from $N_{\mathrm{b}}$ :

$P_{\mathrm{b}}=\frac{N-N_{\mathrm{b}}}{N-2}$,

in which $N$ is the number of walkers and $N_{\mathrm{b}}$ is the number of boundaries. $N_{\mathrm{b}}$ and $N$ are equal in a superlattice or totally mixed distribution $\left(P_{\mathrm{b}}=0\right)$. Note that this result is independent of the spacing (or density) of particles. In a random system, $N_{\mathrm{b}}$ is of the order of $N$, leading to small finite valucs of $P_{\mathrm{b}}$. Total segregation results in a system with two clusters and consequently two boundaries; the resulting value of $P_{\mathrm{b}}$ is $1 . P_{\mathrm{b}}$ is plotted as a function of time for a $1-\mathrm{D}$ system in fig. 1. $P_{\mathrm{b}}$ reaches its steady-state value somewhat earlier than $P_{\mathrm{NN}}$, but the steady-state value for the one-dimensional system is also approximately 0.9 .

The notion of a cluster and associated boundaries is straightforward in one-dimensional systems. Onedimensional clusters can be defined without regard to inter-particle separations and therefore density. In higher dimensions and fractals, the situation is more complicated. Clusters can be readily defined in terms of a specific maximum intra-cluster particle separation, but the appropriate separation depends upon density. We have therefore turned to another feature of particle distributions to obtain parameters by which to describe the global segregation in systems of one dimension and higher: inter-particle separations.

A consequence of the formation of clusters is that particles of the same type, grouped into the same clusters, are more closely spaced than particles of different types, which are in different clusters. In order to characterize these separations, we have extended the nearest (adjacent) neighbor concept of section 3.1 to a "closest" neighbor concept. The closest neighbor to a particle is the particle at the shortest distance, where distance is defined by taxicab geometry (illustrated in fig. 2b). By counting the number of particles in a system with a closest neighbor at a given distance $r$, we obtain a distribution of closest neighbor separations, designated $P_{\mathrm{CN}}(r)$. Through the use of the taxicab definition of distance on discrete, disordered or fractal lattices, $P_{\mathrm{CN}}(r)$ can be used to characterize particle distributions in arbitrary topologies. We note that this definition is analogous to the "Hertz nearest (closest) neighbor" distribution $[15,16]$.

$P_{\mathrm{CN}}(r)$ is calculated from a series of breadth-first searches [17], one for each particle. For each particle, the set of sites within one lattice unit $(r=1)$ is identified. If any is occupied, $P_{\mathrm{CN}}(1)$ is incremented by 1 . If not, the set of $r=2$ sites is formed from the nearest neighbors of the $r=1$ sites, excluding those which have already been examined. If no $r=2$ site is occupied, the process is repeated until an occupied site is encountered in the set of $r=n$ sites. $P_{\mathrm{CN}}(n)$ is then incremented by 1 . This straightforward breadth- 
first search method is appropriate for any lattice with a single lattice spacing. More sophisticated techniques are required for lattices with unequal spacings between adjacent sites.

$P_{\mathrm{CN}}(r)$ may be determined separately for matched and mixed pairs in order to cxaminc the difference in separations of like and unlike particles. The closest neighbor separations of matched pairs reflect intra-cluster particle separations and therefore intracluster densities. The closest neighbor separations of mixed pairs, on the other hand, are inter-cluster distances by definition. They therefore reflect the distances between adjacent clusters of different particle types. The greater the difference in the most probable (or average) closest neighbor separation of mixed and matched pairs, the greater the extent of clusterization and hence of segregation. Corrections for density effects on each of the $P_{\mathrm{CN}}(r)$ can be made by scaling $r$ by its most probable value.

$P_{\mathrm{CN}}(r)$ is presented in fig. 3 for matched ("AA") and mixed ("AB") pairs at steady state on a one-dimensional lattice. For comparison, the curve obtained from a random distribution of the same density is also shown. As expected, the most probable separation of matched pairs is significantly smaller

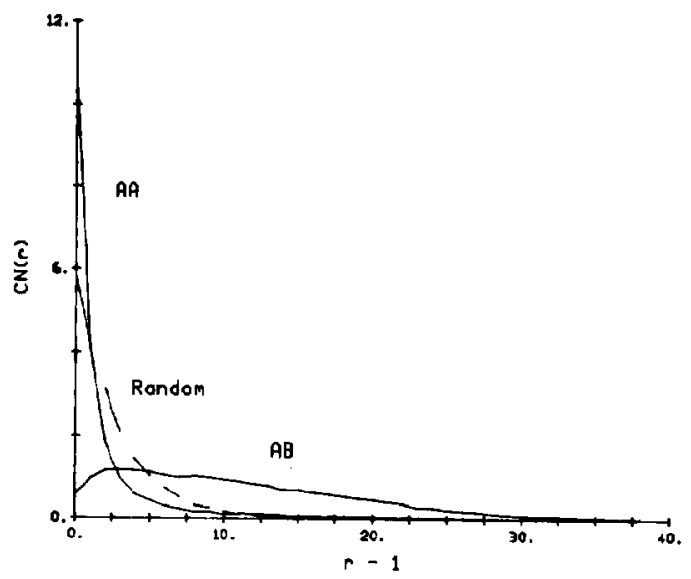

Fig. 3. Steady-state closest neighbor distributions - 1-D. $P_{\mathrm{CN}}(r)$ is plotted as a function of $r-1$ for the 1-D lattice at (or near) steady state. AA: matched pairs: AB: mixed pairs. Results for random distributions (dashed line) are included for comparison. The simulation curves represent averages over 50 time points from each of 10 runs. Two walkers were added per step to a 128 site lattice (with vertical annihilation and cyclic boundary conditions). than that of the random distribution, due to increased intra-cluster densities. The mixed pair distribution is nearly flat. This arises from the relatively even spacing of particles within one cluster. The distances from the boundary, the closest point at which a particle of the other type may be found, to each particle in a cluster are fairly evenly distributed. $P_{\mathrm{CN}}(r)$ for matched and mixed pairs at steady state on the Sierpinski gasket is shown in fig. 4 and seen to have qualitatively similar features to those of the 1-D lattice (fig. 3), except that the random curve is not a decaying exponential but qualitatively similar to a two-dimensional Hertz distribution [15,16], as expected [18].

Distributions such as those of figs. 3 and 4 are useful for characterizing particle distributions at a particular time. A single parameter, however, is more convenient for monitoring the development of segregation over time, or for quantitative comparisons between systems of different topologies. One such parameter is given by

$P_{\langle\mathrm{CN}\rangle}=1-\frac{\left\langle R_{\mathrm{CN}}(\mathrm{AA})\right\rangle}{\left\langle R_{\mathrm{CN}}(\mathrm{AB})\right\rangle}$

in which $\left\langle R_{\mathrm{CN}}(\mathrm{AA})\right\rangle$ and $\left\langle R_{\mathrm{CN}}(\mathrm{AB})\right\rangle$ are the average closest neighbor separations of matched and

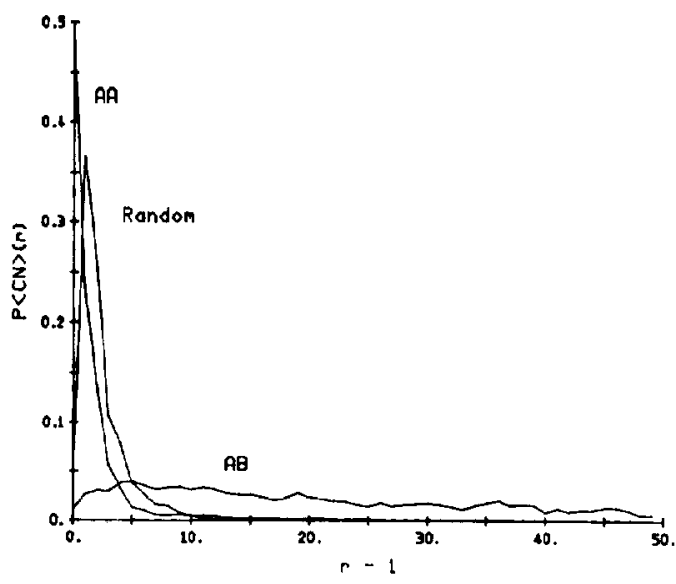

Fig. 4. Steady-state closest neighbor distributions - Sicrpinski gasket. $P_{C N}(r)$ is plotted as a function of $r-1$ for the Sierpinski gasket at steady state ( $10^{6}$ time steps). AA: matched pairs; $A B$ mixed pairs. The curves represent averages over 2 runs. Sixteen walkers were added per step to an eight-order Sierpinski gasket (without vertical annihilation). For further details see ref. [5]. 


$$
P_{\langle C N\rangle}=1-\left\langle R_{A B}\right\rangle /\left\langle R_{A B}\right\rangle
$$

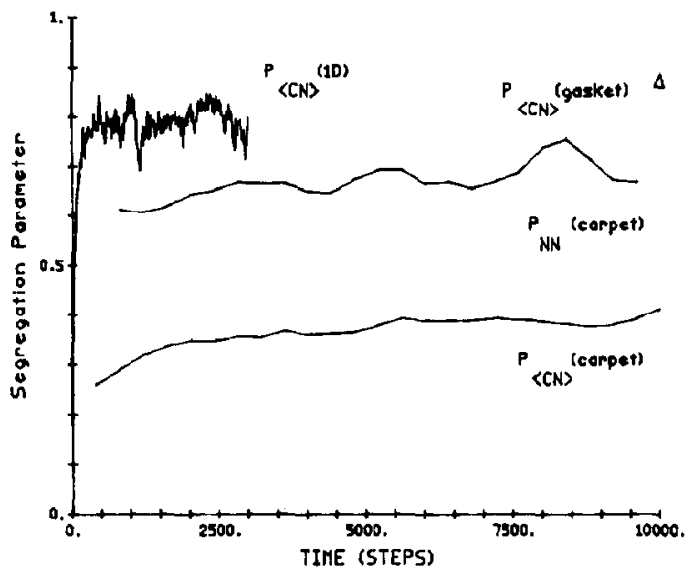

Fig. 5. Closest neighbor segregation parameter, $P_{\langle\mathrm{CN}\rangle}$ (eq. (3)) is plotted as a function of time for the 1-D lattice and the Sierpinski carpet. The steady-state value for the Sierpinski gasket is plotted as a single point in the upper left. $P_{\mathrm{NN}}$ for the Sierpinski carpet is included for comparison. For further details see captions of figs. 3 and 4 and ref. [10].

mixed pairs, respectively. In a random system, these two quantities are equal and $P_{\langle\mathrm{CN}\rangle}$ is 0 . In a totally segregated system of infinite extent, $\left\langle R_{\mathrm{CN}}(\mathrm{AB})\right\rangle$ goes to infinity, the ratio to 0 , and $P_{\langle\mathrm{CN}\rangle}$ to 1 . For finite systems, values of 1 cannot be achieved, but values on the order of $1-1 / L$, where $L$ is the lattice size, are expected. Since $\left\langle R_{\mathrm{CN}}(\mathrm{AA})\right\rangle$ depends upon the overall particle density, the ratio can be corrected for density for quantitative comparisons of segregation in systems with significantly different densities.

$P_{\langle\mathrm{CN}\rangle}$ is plotted as a function of time for steadystate simulations on the 1-D lattice and Sierpinski carpet in fig. 5 . The steady-state value after $10^{6}$ steps obtained from particle distributions on the Sierpinski gasket is included as a single point. For comparison, the parameter $P_{\mathrm{NN}}$ (eq. (1)) for the Sierpinski carpet is also plotted. Although the magnitudes of $P_{\langle\mathrm{CN}\rangle}$ and $P_{\mathrm{NN}}$ differ, their time dependence is quite similar.

\section{Discussion}

The kinds of quantitative measures required to establish relationships between topology, reactant segregation and reaction kinetics are those which are gencralizable to low and high densitics, and to all lattice topologies. Three parameters have been presented here, each with certain strengths and weaknesses.

The parameter which is sensitive to local effects, $\Gamma_{\mathrm{NN}}$, can be extended to any dimensionality (Euclidean or fractal). It is, however, limited to systems with moderate or high particle densities, because it relies on the abundance of pairs of particles at adjacent sites. Both of the global segregation parameters defined here are valid at any particle density. $P_{\mathrm{b}}$ is limited by lattice topology, because cluster boundaries are not easily defined in higher-dimensional or fractal lattices. The third parameter, $P_{\langle\mathrm{CN}\rangle}$, is readily applied to any lattice, as long as a taxicab definition of distance is employed.

All three parameters have well-defined limits: 0 for random or perfectly mixed systems and 1 for complete segregation. $P_{\mathrm{NN}}$ breaks down in super-lattice structures of densities below 1 , for the same reason that it is not usable at low densities in general. The theoretical upper limit of $P_{\langle\mathrm{CN}\rangle}$ can only be achicved in systems of infinite extent. Corrections for finite lattice size are needed before quantitatively comparing segregation on lattices of different sizes. In addition, $P_{\langle\mathrm{CN}\rangle}$ retains some density dependence, which can also be corrected.

Criteria for consistent evaluation of reactant segregation in a wide variety of systems are essential for understanding the relationship between segregation and reaction kinetics. We note that Hertz distribution functions [18-20] which are analogous to $P_{\mathrm{CN}}(r)$ have been related quantitatively to reaction rate laws. This relationship has not been established for the $A+B$ reaction, but it is obvious from our work that the kinetics and segregation measures are interrelated. Parameters which can be generalized to arbitrary geometries and densities will prove of most value in the development of an integrated view of reaction kinetics in low-dimensional systems. The particular measures of segregation suggested here are not the only ones possible. Other segregation paramcters $[7,8]$, along similar as wcll as markedly dif- 
ferent lines, can, and certainly should, bc investigated.

\section{Acknowledgement}

We thank Eric Clement and Dr. Stephen J. Parus for helpful discussions. This research is supported by National Science Foundation Grant No. DMR 8801120.

\section{References}

[1] A.A. Ovchinnikov and Ya.B. Zeldovich, Chem. Phys. 28 (1978) 215 , and references therein.

[2] P.G. de Gennes, J. Chem. Phys. 76 (1982) 3316.

[3] D. Toussaint and F. Wilczek, J. Chem. Phys. 78 (1983) 2642.

[4] A. Blumen, G. Zumofen and J. Klafter, J. Phys. (Paris) 46 (1985) C7-3;

A. Blumen, J. Klafter and G. Zumofen, in: Optical spectroscopy of glasses, ed. I. Zschokke (Reidel, Dordrecht, 1986).

[5] L.W. Anacker and R. Kopelman, Phys. Rev. Letters 58 (1987) 289; J. Phys. Chem. 91 (1987) 5555.

[6] L.A. Harmon, L. Li and R. Kopelman, Bull. Am. Phys. Soc. 32 (1987) 627.

[7] J. Newhouse and R. Kopelman, J. Phys. Chem. 92 (1988) 1538 .
[8] K. Lindenberg, B.J. West and R. Kopelman, Phys. Rev. Letters 60 (1988) 1777.

[9] E. Clement, L. Li, L.W. Anacker, L.A. Harmon, R. Kopelman and L.M. Sander, in: Fractal aspects of materials: disordered systems, eds. D.A. Weitz, L.M. Sander and B.B. Mandelbrot (Materials Research Society, Pittsburgh, 1988) p. 307.

[10] L. Li and R. Kopelman, J. Luminescence 40/41 (1988) 688.

[11 ] L. Li, Ph.D. Thesis, University of Michigan (1989).

[12] E. Clement, L.M. Sander and R. Kopelman, Phys. Rev. A, in press.

[13] L.A. Harmon, L. Li, L.W. Anacker and R. Kopelman, in: Fractal aspects of materials: disordered systems, eds. D.A. Weitz, L.M. Sander and B.B. Mandelbrot (Materials Research Society, Pittsburgh, 1988) p. 303.

[14] J. Hoshen, R. Kopclman and E.M. Monberg, J. Stat. Phys. 19 (1978) 219.

[15] P. Hertz, Math. Ann. 67 (1909) 387.

[16] E.W. Montroll and W.W. Badger, Introduction to quantitative aspects of social phenomena (Gordon and Breach, New York, 1974) p. 100.

[17]E. Horowitz and S. Sahni, Fundanentals of computer algorithms (Computer Science Press, Potomac, 1978).

[18] L. Li, E. Clement, P. Argyrakis, L.A. Harmon, S.J. Parus and $R$. Kopelman, in: Fractal aspects of materials: disordered systems, eds. D.A. Weitz, L.M. Sander and B.B. Mandelbrot (Materials Research Society, Pittsburgh, 1988) p. 211.

[19] R. Kopelman, Science 241 (1988) 1620.

[20] G. Weiss, R. Kopelman and S. Havlin, Phys. Rev. A 39 (1989) 466. 